\title{
Influence de la rotation culturale avec apport de matières organiques exogènes et d'une fertilisation minérale sur les nématodes phytoparasites en culture du sorgho au Centre Ouest du Burkina Faso
}

\author{
Mamoudou TRAORE ${ }^{1 *}$, François LOMPO ${ }^{2}$, Bouma THIO $^{3}$, Badiori OUATTARA ${ }^{2}$, \\ Korodjouma OUATTARA ${ }^{4}$ et Michel SEDOGO ${ }^{2}$
}
1 Institut de l'Environnement et de Recherches Agricoles (INERA), Département Production Forestières 03 BP 7047 Ouagadougou 03 Burkina Faso. Tél : 226503340 98. Fax : 22650340271.
2 Institut de l'Environnement et de Recherches Agricoles (INERA), Laboratoire Sol-Eau-Plantes, 04 BP 8645 Ouagadougou 04, Burkina Faso. Tél : 0022650340270 . Fax : 0022650340271. 3 Institut de l'Environnement et de Recherches Agricoles (INERA), Laboratoire de Nématologie, Station de Farako bâ, 01 BP 910 Bobo Dioulasso 01, Burkina Faso. Tel : 22620970960. 4 Institut de l'Environnement et de Recherches Agricoles (INERA), Station de Recherche de Saria, Laboratoire Sol-Eau-Plantes, BP 10 Koudougou, Burkina Faso. Tel : 22650446509
*Auteur correspondant, E-mail : tramadalbela@yahoo.fr

\section{RESUME}

L'infestation du sol et des racines du sorgho (sorghum bicolor (L.) Moench) par les nématodes phytoparasites a été étudiée en 2008 sur un essai agricole à l'Ouest du Burkina Faso. Cet essai agricole est représentatif de la production du sorgho avec utilisation de différentes pratiques agricoles incluant la rotation culturale, l'apport de matières organiques exogènes et une fertilisation minérale. L'objectif était de déterminer l'effet de ces pratiques sur les taux d'infestation du sol et des racines par les nématodes. Les nématodes du sol ont été extraits selon la méthode de «l'élutriateur» de Seinhorst et ceux des racines selon la méthode de «l'asperseur» de Seinhorst. Le sol et les racines du sorgho ont été plus infestés par les nématodes dans la monoculture de sorgho par rapport aux rotations sorgho - niébé et sorgho - coton. Les traitements incluant la matière organique et la fertilisation minérale ont été moins infestés par les nématodes par rapport aux traitements utilisant uniquement une fertilisation minérale. La nématofaune de la jachère a été plus diversifiée que celle du site cultivé et le principal nématode lié au sorgho, $P$. brachyurus, y a fortement régressé.

(C) 2012 International Formulae Group. All rights reserved.

Mots clés : rotation, fertilisation minérale, matière organique, nématodes.

\section{INTRODUCTION}

Le sorgho (Sorghum bicolor (L) Moench) est la cinquième céréale la plus cultivée dans le monde, après le maïs, le blé, le riz et l'orge. Sa production représente 61,7 millions de tonnes en 2007/2008 dont 25,6 millions de tonnes pour l'Afrique où il couvre 4\% des terres arables. Au Burkina Faso, le sorgho est la céréale la plus répandue, sa culture y est pratiquée partout en saison des pluies, là où les précipitations sont supérieures à 400-500 mm. La superficie consacrée à la 
culture du sorgho est passée de 1225223 ha en 2000 à 1983120,30 ha en 2010. Les quantités de céréales produites passent à la même période de 847297 tonnes à 1990227 tonnes (FAO, 2010). Malgré cette hausse de rendement, le Burkina Faso, à l'instar de la plupart des pays du Sahel, est confronté depuis de nombreuses années à une grave crise alimentaire liée aux effets des phases de sécheresse mais aussi à la dégradation de la qualité des terres cultivables (Sedogo, 1993 ; Ouédraogo, 2005 ; Lompo, 2008). La culture du sorgho, comme toutes les autres cultures vivrières du Burkina Faso, est aussi fortement tributaire des conditions climatiques dont les fluctuations affectent considérablement la production d'ensemble. A côté de ces aléas climatiques, l'inadéquation des pratiques culturales, la pauvreté des sols et la faible fertilisation sont soulignées comme les contraintes majeures à la production agricole au Burkina Faso (Sedogo, 1993 ; Bado, 2002 ; Bonzi, 2002 ; Hien, 2004). En particulier, une fertilisation inadéquate ne permet pas aux plantes de lutter efficacement contre les maladies et ravageurs, qui constituent de principales contraintes à la production agricole.

Ces ravageurs et maladies des céréales ont été reconnus depuis longtemps comme des contraintes importantes à la production agricole dans le monde entier et ont été l'objet de recherches approfondies. Parmi les facteurs biotiques, les nématodes phytoparasites pourraient constituer l'un des principaux organismes impliqués dans la perte de récolte des cultures céréalières au champ (Weber et al., 1994; Mc Donald et al., 2005). Cependant, de tous les dangers potentiels qui menacent les cultures, les nématodes sont souvent les moins connus et ont été en grande partie exclus de l'attention de la recherche (Talwana et al., 2008). Les données sur l'importance des nématodes, la composition et la densité des populations, leur caractère pathogène, en particulier la survenue des dommages causés par certaines espèces ou groupes d'espèces, sont peu nombreuses
(Cadet, 1998 ; Bois et al., 2000). Dans la zone sahélienne, cette méconnaissance est aussi consécutive au fait que relativement peu d'études nématologiques y ont été conduites en comparaison de son immensité et de la diversité des sols ou des systèmes culturaux que l'on y rencontre. Cependant, plusieurs travaux ont démontré l'effet dévastateur des nématodes phytoparasites sur les cultures vivrières dans le monde en général et en Afrique semi-aride en particulier. Ils ont été signalés comme de graves entraves aux productions céréalières et maraîchères dans différentes régions du monde (Bois et al., 2000 ; Bélair, 2005). Ils sont présents sous toutes les latitudes et représentent un grave problème phytosanitaire, surtout dans le monde tropical où règne en permanence un climat favorable à leur multiplication. Ces déprédateurs affectent les rendements des cultures tropicales des pays du Tiers-monde, d'où leur importance économique (Prot, 1985 ; Stoll, 2002 ; Talwana et al., 2008). En Afrique Subsaharienne, la presque totalité des cultures vivrières et de rente enregistrent des baisses de rendements du fait des nématodes qui les parasitent (De Weale et al., 1988; Cadet, 1998). Ces baisses de rendement dues aux nématodes peuvent atteindre 25 à $40 \%$ en l'absence de traitement nématicide (Mc Donald et al., 2005). Pour le sorgho, bien qu'un certain nombre de nématodes qui lui sont associés aient été inventoriés, peu d'informations sont disponibles sur les espèces inféodées à cette culture. Mais l'augmentation des rendements (jusqu'à 70\%) après le traitement chimique du sol où des densités élevées de population de ces nématodes inféodés ont été enregistrées, fournit des preuves indirectes de dommages économiques considérables (Prot, 1985 ; Mc Donald et al., 2005).

L'objectif global de cette étude est d'identifier les nématodes phytoparasites du sol et dans les racines du sorgho sous différentes pratiques culturales (apport de matières organiques, fertilisation minérale et rotation) et de montrer l'influence de ces 
pratiques agricoles sur les populations de nématodes. De manière spécifique, il s'agissait de déterminer la composition et l'importance des espèces de nématodes sous ces différentes pratiques culturales. Cette étude a eu pour hypothèse selon laquelle les pratiques culturales telles que la rotation culturale et l'apport de fertilisations peuvent permettre de réduire l'infestation en nématodes du sorgho.

\section{MATERIEL ET METHODES \\ Description du site}

Cette étude s'est déroulée en 2008 sur un essai agricole situé dans la station de recherches environnementales et agricoles de Saria $\left(12^{\circ} 16^{\prime} \mathrm{N}\right.$ et $\left.2^{\circ} 9^{\prime} \mathrm{W}\right)$ au Centre-Ouest du Burina Faso. La station de recherches de Saria est soumise à un climat de type soudanien caractérisé par l'existence de deux saisons très marquées: une longue saison sèche (octobre à mai) et une courte saison de pluie (juin à octobre). L'altitude de cette station est de $300 \mathrm{~m}$. La température moyenne annuelle est de $28{ }^{\circ} \mathrm{C}$. Les précipitations moyennes annuelles sont de $800 \mathrm{~mm}$ et le sol est classé dans la catégorie des sols ferrugineux tropicaux lessivés (Hien, 2004).

\section{Description du dispositif expérimental}

Le dispositif expérimental a été mis en place en 1960 pour étudier la fertilité d'un sol ferrugineux tropical sous l'influence de différentes pratiques culturales comprenant des fumures minérales et organiques. Trois systèmes de culture sont mis en ouvre: monoculture de sorgho (S), rotation sorghocoton (SC), rotation sorgho-niébé (SN). Le dispositif est un split-plot en blocs complets avec six répétitions. La dimension du site est $161,2 \mathrm{~m}$ x $60 \mathrm{~m}=9672 \mathrm{~m}^{2}$. Les parcelles mesurent $10 \mathrm{~m}$ de long sur une largeur de 8,40 $\mathrm{m}$. Une allée de $2 \mathrm{~m}$ sépare deux blocs voisins. Six traitements identiques sont appliqués à chacun des trois systèmes de culture:

T1 : témoin absolu ;
T2: e + $\mathbf{r}$ : fumure minérale faible (37-23-14$6 S-1 B)+$ recyclage des pailles de sorgho tous les deux ans ;

T3: $\mathbf{e}+\mathbf{f}$ : fumure minérale faible + fumier $(5$ t.ha ${ }^{-1} \cdot 2$ ans $\left.^{-1}\right)+$ exportation des pailles de sorgho. Il convient de noter que jusqu'en 1976, le fumier est apporté annuellement en raison de 5 t. ha ${ }^{-1}$;

T4: e : fumure minérale faible sans fumier; T5: E + F : fumure minérale forte (60-23-44$6 \mathrm{~S}-1 \mathrm{~B})+$ fumier $\left(40 \mathrm{t}^{-h^{-1}} \cdot 2 \mathrm{ans}^{-1}\right)+$ exportation des pailles de sorgho; de 1960 à 1963, le fumier est apporté à raison de 5 t.ha ${ }^{1}$. an ${ }^{-1}$. De 1964 à 1976, la dose passe à 40 t.M $\mathrm{ha}^{-1} \cdot \mathrm{an}^{-1}$. C'est après 1976 donc que les doses actuelles de $40 \mathrm{t}$ sont adoptées;

T6: E : fumure minérale forte sans fumier.

Le recyclage de la paille sur une parcelle est effectué à partir des pailles produites sur la même parcelle l'année précédente. La paille est hachée avant d'être apportée sur la parcelle. Le fumier est un mélange de paille, de sorgho et de déjections animales produites à l'étable, stocké dans des fosses où il évolue pendant six mois environ. Le fumier et la paille sont enfouis par le labour ainsi que les engrais minéraux lors du semis. Les engrais apportés à la montaison et à la floraison sont enfouis par le sarclobinage réalisé juste après leur épandage. Les engrais apportés au semis sont le chlorure de sodium $(\mathrm{KCl})$ et le NPK à raison respectivement de 50 et $100 \mathrm{~kg} / \mathrm{ha}$. L'apport de l'urée est fractionné, $50 \%$ à la montaison et $50 \%$ à la floraison.

\section{Échantillonnage des nématodes}

Les nématodes ont été échantillonnés selon la méthode des cultures en lignes. L'échantillon d'une parcelle est constitué par des prélèvements de sols effectués à $20 \mathrm{~cm}$ de profondeur en plusieurs endroits à l'aide d'une truelle, en fonction de la taille de la parcelle, de manière à couvrir toute la parcelle (pour la taille de nos parcelles, le prélèvement a été fait en 5 points). Un échantillon pèse en moyenne $3 \mathrm{~kg}$. Le prélèvement des échantillons a été effectué au lendemain de la 
récolte. Les populations de la plupart des nématodes phytoparasites dans le sol ont tendance à culminer en cette période, une fois que la partie aérienne des cultures s'est fanée ou morte (Celetti, 2006). Au total, 54 échantillons ont été prélevés sur le site d'étude et 03 échantillons dans la jachère en bordure du site. Les échantillons de sols ont été placés dans de sacs plastiques attachés et étiquetés ; placés à l'abri des rayons du soleil dans un endroit frais et transportés vers le laboratoire de nématologie du Centre Régional de Recherches Environnementales et Agricoles (CRREA) de Farako-bâ (Bobo Dioulasso, Burkina Faso) pour l'extraction et l'identification des nématodes.

\section{Extraction des nématodes}

L'extraction des nématodes a été faite en utilisant la méthode de « l'élutriateur » et de «l'asperseur » de Seinhorst respectivement pour les nématodes du sol et des racines (Merny et al., 1969). Pour les nématodes du sol, une fraction de sol de $250 \mathrm{~cm}^{3}$ a été prélevée par échantillon et soumis aux trois phases que comporte cette méthode d'extraction: la phase d'élutriation, la phase de tamisage et la phase de filtration active qui sépare les nématodes des impuretés du sol sur la base de leur mobilité. Après extraction, le dénombrement des nématodes est effectué à l'aide de la plaque de comptage sous la loupe binoculaire. Les effectifs de la population sont exprimés en nématodes $/ \mathrm{dm}^{3}$ de $\operatorname{sol}\left(\mathrm{N} / \mathrm{dm}^{3}\right)$.

Pour les nématodes des racines, l'échantillon des racines est lavé, découpé en menus morceaux, placé sur un support à grosses mailles et déposé dans un entonnoir relié à un récipient. Les racines sont maintenues sous un brouillard pendant 14 jours. Leur décomposition entraîne la libération des nématodes qui sont retenus dans le récipient. Ils sont soumis à une filtration active avant le comptage. Après extraction, le dénombrement des nématodes est effectué à l'aide de la plaque de comptage sous la loupe binoculaire. Les effectifs de la population des nématodes sont exprimés en nématodes/ gramme de racines fraîches (N/g racines).

\section{Analyse des données}

Les données obtenues ont été introduites dans le tableur Excel 2003 et soumises à l'analyse de variance (ANOVA), General Linear Model (GLM) en utilisant Minitab version 13.0. La matrice d'analyse est un "full rank" constitué des facteurs (type de culture et type de traitements), et des variables (constituées par les espèces de nématodes identifiées). Les moyennes ont été comparées en utilisant le test de Fisher au seuil de probabilité $5 \%$.

\section{RESULTATS}

\section{Les nématodes du sol}

Notre étude a identifié 05 espèces de nématodes sur le site de culture et 07 espèces dans la jachère. Il s'agit de Pratylenchus brachyurus Godfrey, 1929 (Famille : Hoplolaimidae, sous-famille : Pratylenchinae), Tylenchorhynchus martini Cobb, 1913 (Famille: Tylenchidae), Helicotylenchus multicinctus Cobb, 1893 (Famille Hoplolaimidae), Scutellonema cavenessi Andrassy, 1958 (Famille Hoplolaimidae), Criconemö̈des curvatum Raski, 1952 (Famille: Criconematidae), Telotylenchus indicus Siddiqi, 1960 (Famille : Tylenchidae), et Xiphinema sp Cobb, 1913 (Famille: Dorylaimidae). Leurs densités varient de 0 à 3220 par dm $^{3}$ de sol.

Trois (03) espèces ( $P$. brachyurus, $T$. martini et $H$. multicinctus) représentent plus de $98 \%$ du nombre total de nématodes. Les deux autres nématodes $S$. cavenessi et $C$. curvatum sont très faiblement représentés à raison de 0 à 60 individus par $\mathrm{dm}^{3}$ de sol (Tableau 1). La densité de population de $P$. brachyurus est significativement corrélée au type de rotation $(\mathrm{p}=0,002)$ et non significative quant au type de traitement $(\mathrm{p}=$ 0,731). La densité de population de T. martini est significativement corrélée au type de traitement $(\mathrm{p}=0,007)$ et non significative pour le type de rotation $(\mathrm{p}=0,892)$; celle de 
H. multicinctus est significativement corrélée au type de rotation $(\mathrm{p}=0,001)$ et au type de traitement $(\mathrm{p}=0,034)$. Les densités de population de $S$. cavenessi et $C$. curvatum ne sont significativement corrélées ni au type de rotation ( $p=1,000$ et $\mathrm{p}=0,376)$ ni au type de traitement $(p=0,273$ et $p=0,428)$.

Dans la monoculture de sorgho (S), nous enregistrons de faibles infestations en nématodes du sol avec les traitements avec utilisation de matières organiques et la fertilisation minérale (T3, T5) et le traitement avec une forte dose de fumure minérale (T6) (Figure 1). Les deux principaux nématodes, $P$. brachyurus et $T$. martini, enregistrent des infestations respectives de plus de 3000 $\mathrm{N} / \mathrm{dm}^{3}$ pour le traitement T2. Pour le traitement $\mathrm{T} 4$, leurs taux d'infestation atteignent respectivement plus de 3000 et $1000 \mathrm{~N} / \mathrm{dm}^{3}$ de sol. Les deux autres nématodes ont de très faibles infestations, de 0 à $200 \mathrm{~N} / \mathrm{dm}^{3}$ de sol. Dans la rotation sorghocoton, les infestations sont dues essentiellement à $T$. martini et $H$. multicinctus avec des densités de population atteignant $2827 \mathrm{~N} / \mathrm{dm}^{3}$ de sol au traitement T2 (Figure 2). Les deux autres espèces $P$. brachyurus et $S$. cavenessi apparaissent avec des densités de population très faibles, de 0 à $80 \mathrm{~N} / \mathrm{dm}^{3}$ de sol. Les traitements T3, T5 et T6 sont les traitements les moins infestés en nématodes du sol. La rotation sorgho-niébé enregistre une infestation en nématodes du sol due aussi essentiellement à $T$ martini et $H$ multicinctus (Figure 3). Leurs densités de population sont par contre plus importantes qu'au niveau du sorgho-coton. On enregistre jusqu'à 3770 $\mathrm{N} / \mathrm{dm}^{3}$ de sol pour T. martini sous le traitement T2. L'espèce $P$. brachyurus qui avait pratiquement disparue sous la rotation sorgho-coton réapparaît ici avec des densités de population allant de 13 à $1187 \mathrm{~N} / \mathrm{dm}^{3}$ de sol sous T6. Les traitements T3, T4 et T5 sont sous cette rotation les traitements les moins infestés. De façon générale, la rotation sorgho continu est la plus infestée en nématodes, toute espèce confondue suivie de la rotation sorgho-coton (Tableau 2). Ces deux rotations représentent respectivement $44 \%$ et $35 \%$ du total des infestations contre $21 \%$ pour la rotation sorgho-niébé.

Les différents traitements se sont comportés différemment en termes de taux d'infestation. Dans toutes les rotations, les traitements T2 et T4 qui associent la fumure minérale de faible dose sans fumier ont été les plus infestés (Tableau 3). Ces deux traitements ont représenté en moyenne 55\% des infestations pour le sorgho continu et le sorgho-coton. Pour la rotation sorgho-niébé, le traitement T6 rejoint les traitements les plus infestés et les trois traitements regroupent $77 \%$ des infestations. Les traitements associant la fumure minérale et le fumier (T3, T5) se sont révélés par contre moins infestés par les nématodes. Pour toute espèce confondue, ces traitements entraînent en moyenne $10 \%$ des infestations par rotation.

Dans la jachère, on note une meilleure représentation d'espèces qui avaient des densités de population très faibles sur les sites de culture (Figure 4). P. brachyurus, qui avait des populations plus importantes sur les sites de culture régresse dans la jachère avec une densité maximale de $630 \mathrm{~N} / \mathrm{dm}^{3}$ de sol. $T$. martini et $H$. multicinctus sont alors les deux espèces les mieux représentées dans la jachère.

\section{Les nématodes des racines}

$P$. brachuyrus, nématode des lésions racinaires, a été le seul nématode retrouvé dans les racines du sorgho avec des taux d'infestation allant de 0 à $35 \mathrm{~N} / \mathrm{g}$ racine (Tableau 4). Dans la rotation sorgho continu, le traitement témoin $\mathrm{T} 1$ est le plus infesté et le T5 (fumure minérale forte + fumier), le moins infesté. Le taux moyen d'infestation sur ce site est $12 \mathrm{~N} / \mathrm{g}$ racines en prenant en compte tous les traitements. Au niveau de la rotation sorgho-coton, les taux d'infestation vont de 0 à $18 \mathrm{~N} / \mathrm{g}$ racines. De manière générale, exception faite du traitement $\mathrm{T} 1$, les infestations sont faibles pour les autres traitements. Le taux d'infestation moyen au niveau de cette rotation est $5,5 \mathrm{~N} / \mathrm{g}$ racines. $\mathrm{La}$ 
même tendance s'observe au niveau de la rotation sorgho-niébé avec un taux de $11 \mathrm{~N} / \mathrm{g}$ racines pour le $\mathrm{T} 1$ et des infestations de 0 à 4 pour les 05 autres traitements. Le taux moyen d'infestation de la rotation sorgho-niébé est de $3,5 \mathrm{~N} / \mathrm{g}$ racines. Les racines des cultures sont plus infestées par $P$. brachuyrus dans la rotation sorgho continu par rapport aux deux autres types de rotation, sorgho-coton et rotation sorgho-niébé. Le sorgho-niébé étant la rotation où les racines des plantes sont les moins infestées par $P$. brachuyrus. Les résultats montrent une très faible présence de $P$. brachuyrus dans la jachère, 01 nématode par gramme de racines.

Tableau 1: Infestation en nématodes des différentes rotations en fonction des traitements $\left(\mathrm{N} / \mathrm{dm}^{3} \mathrm{de} \mathrm{sol}\right)$.

\begin{tabular}{|c|c|c|c|c|c|}
\hline & Praty & Tylencho & Helico & Scutello & Crico \\
\hline ST1 & $2433 \pm 191$ & $1320 \pm 105$ & $113 \pm 8,72$ & 0 & 0 \\
\hline ST2 & $3591 \pm 287$ & $2963 \pm 239$ & $27 \pm 2,21$ & $60 \pm 5$ & 0 \\
\hline ST3 & $940 \pm 72$ & $727 \pm 61$ & $80 \pm 6,47$ & 0 & 0 \\
\hline ST4 & $3280 \pm 263$ & $1207 \pm 96$ & $27 \pm 2,19$ & 0 & 0 \\
\hline ST5 & $460 \pm 32$ & $353 \pm 32$ & $200 \pm 16,01$ & 0 & 0 \\
\hline ST6 & $1175 \pm 94$ & $1027 \pm 82$ & 0 & 0 & 0 \\
\hline SCT1 & $20 \pm 1,54$ & $707 \pm 55$ & $287 \pm 25$ & $20 \pm 1,63$ & 0 \\
\hline SCT2 & $20 \pm 1,49$ & $2827 \pm 228$ & $193 \pm 17$ & $13 \pm 1,62$ & 0 \\
\hline SCT3 & $80 \pm 6,1$ & $853 \pm 71$ & $260 \pm 20$ & $10 \pm 0,85$ & 0 \\
\hline SCT4 & $13 \pm 1,02$ & $980 \pm 77$ & $1220 \pm 102$ & 0 & 0 \\
\hline SCT5 & $7 \pm 0,53$ & $1320 \pm 106$ & $227 \pm 10$ & $20 \pm 1$ & 0 \\
\hline SCT6 & $27 \pm 2,24$ & $520 \pm 40$ & $27 \pm 2,2$ & 0 & 0 \\
\hline SNT1 & $20 \pm 1,52$ & $1153 \pm 92$ & $100 \pm 8,24$ & $20 \pm 1,4$ & 0 \\
\hline SNT2 & $13 \pm 1$ & $3770 \pm 305$ & $667 \pm 51$ & $13 \pm 0,1$ & $27 \pm 2,6$ \\
\hline SNT3 & $120 \pm 9,88$ & $1067 \pm 85$ & $520 \pm 38$ & 0 & 0 \\
\hline SNT4 & $220 \pm 17$ & $623 \pm 50$ & $1307 \pm 103$ & $20 \pm 1,09$ & 0 \\
\hline SNT5 & $113 \pm 9,23$ & $527 \pm 41$ & $127 \pm 10$ & $7 \pm 0,05$ & 0 \\
\hline SNT6 & $1187 \pm 95$ & $967 \pm 77$ & $3220 \pm 262$ & 0 & 0 \\
\hline$P$ value : Type de rotation & $0,002^{*}$ & 0,892 & $0,001^{*}$ & 1,000 & 0,376 \\
\hline$P$ value : Type de traitement & 0,731 & $0,007^{*}$ & $\mathbf{0 , 0 3 4}$ & 0,273 & 0,428 \\
\hline
\end{tabular}

Tableau 2: Nombre de nématodes $\left(\mathrm{N} / \mathrm{dm}^{3}\right.$ de sol) par rotation.

\begin{tabular}{lcc}
\hline Type de rotation & Nombre de nématodes & Pourcentage \\
\hline S & 19823 & $44 \%$ \\
SC & 15718 & $35 \%$ \\
SN & 9523 & $21 \%$ \\
\hline
\end{tabular}


Tableau 3: Importance relative de l'infestation en nématodes (toute espèce confondue) des différentes rotations en fonction du traitement appliqué.

\begin{tabular}{llll}
\hline & S & SC & SN \\
\hline T1 & $19 \%$ & $11 \%$ & $8 \%$ \\
T2 & $\mathbf{3 3 \%}$ & $\mathbf{3 2 \%}$ & $\mathbf{2 8 \%}$ \\
T3 & $9 \%$ & $12 \%$ & $11 \%$ \\
T4 & $\mathbf{2 5 \%}$ & $\mathbf{2 3 \%}$ & $\mathbf{1 4 \%}$ \\
T5 & $5 \%$ & $5 \%$ & $5 \%$ \\
T6 & $11 \%$ & $6 \%$ & $\mathbf{3 5 \%}$ \\
\hline
\end{tabular}

Tableau 4: Infestation des racines en nématodes des différentes rotations en fonction des traitements appliqués.

\begin{tabular}{lc}
\hline Traitements appliqués aux rotations & Nombre de nématodes / g de racines \\
\hline ST1 & $35 \pm 2,8$ \\
ST2 & $8 \pm 0,56$ \\
ST3 & $4 \pm 0,29$ \\
ST4 & $12 \pm 0,90$ \\
ST5 & $2 \pm 0,14$ \\
ST6 & $11 \pm 0,79$ \\
SCT1 & $18 \pm 1,24$ \\
SCT2 & $6 \pm 0,43$ \\
SCT3 & $1 \pm 0,07$ \\
SCT4 & $3 \pm 0,22$ \\
SCT5 & $0 \pm 0,0$ \\
SCT6 & $5 \pm 0,36$ \\
SNT1 & $11 \pm 0,80$ \\
SNT2 & $4 \pm 0,29$ \\
SNT3 & $0 \pm 0,00$ \\
SNT4 & $1 \pm 0,07$ \\
SNT5 & $3 \pm 0,22$ \\
SNT6 & $2 \pm 0,14$ \\
Jachère & $1 \pm 0,067$ \\
\hline ST1 ST6
\end{tabular}

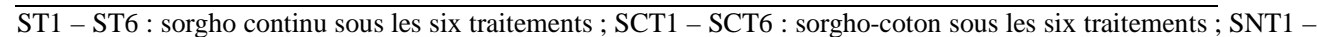
SNT6 : sorgho-niébé sous les six traitements. 


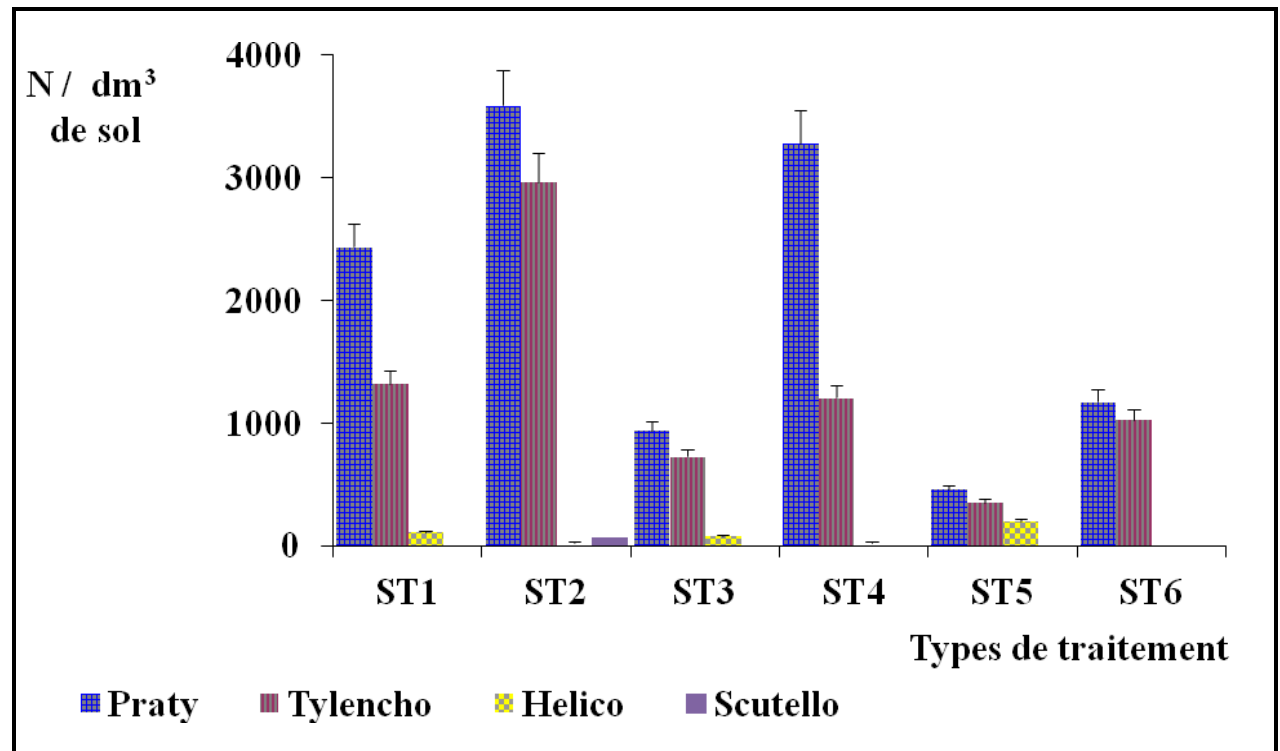

Praty $=$ P. brachyurus $;$ Tylencho $=T$. martini $;$ Hélico $=H$. multicinctus $;$ Scute $=S$. cavenessi .

Figure 1 : Infestation en nématodes de la rotation Sorgho continu (S) sous l'influence des différents traitements (T1 - T6).

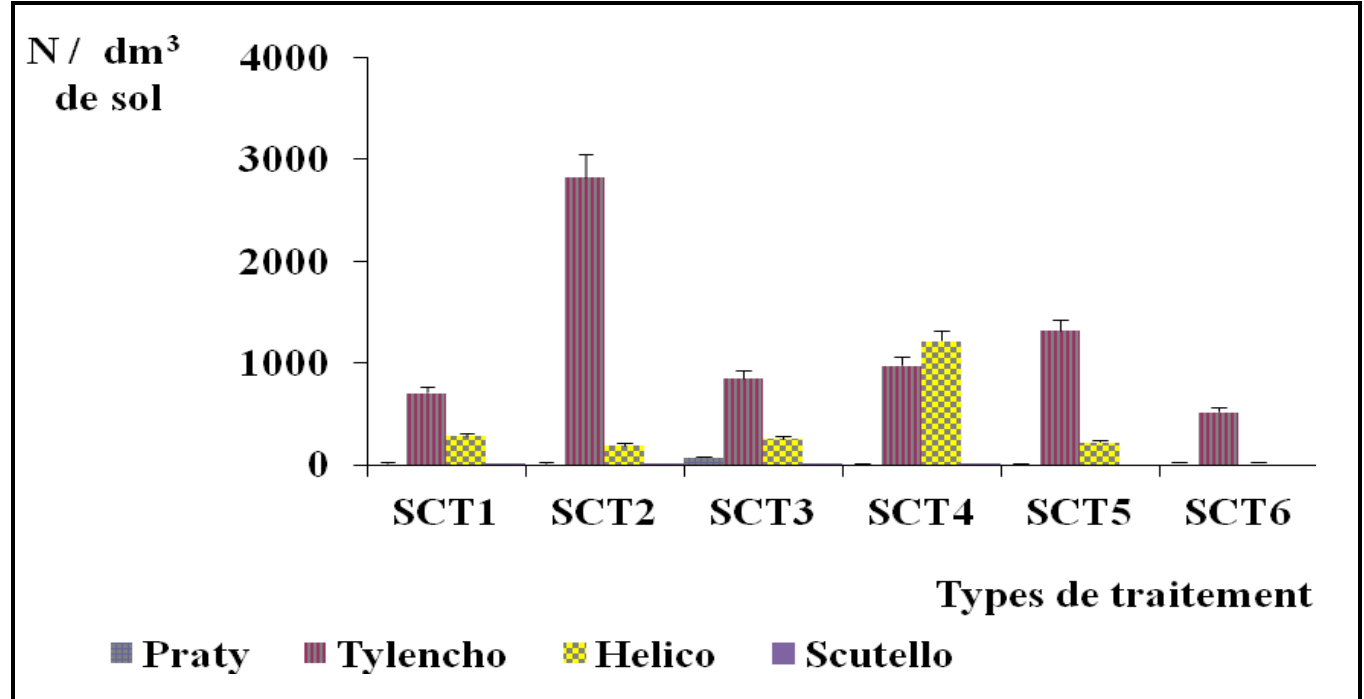

Praty $=$ P. brachyurus $;$ Tylencho $=T$. martini $;$ Hélico $=H$. multicinctus $;$ Scute $=S$. caveness $i$

Figure 2 : Infestation en nématodes de la rotation Sorgho-Coton (SC) sous l'influence des différents traitements $(\mathrm{T} 1-\mathrm{T} 6)$. 


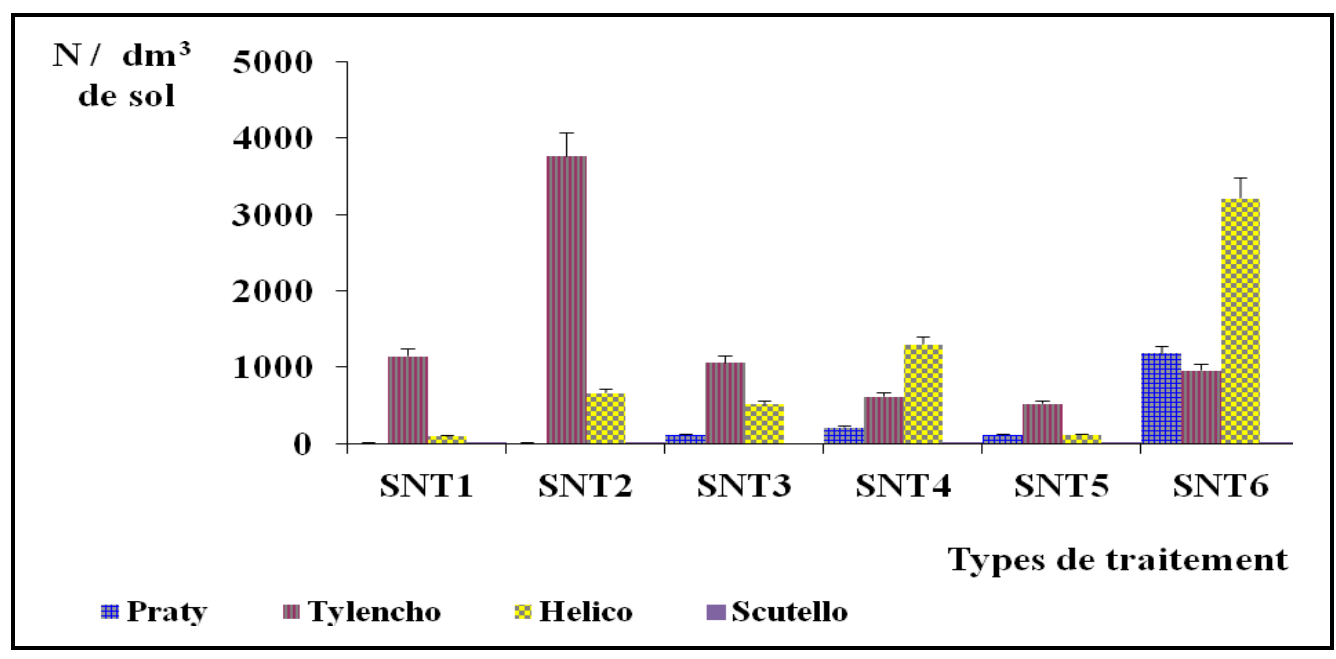

Praty = P. brachyurus $;$ Tylencho $=$ T. martini $;$ Hélico $=H$. multicinctus $;$ Scute $=S$. cavenessi.

Figure 3 : Infestation en nématodes de la rotation Sorgho Niébé (SN) sous l'influence des différents traitements (T1-T6).

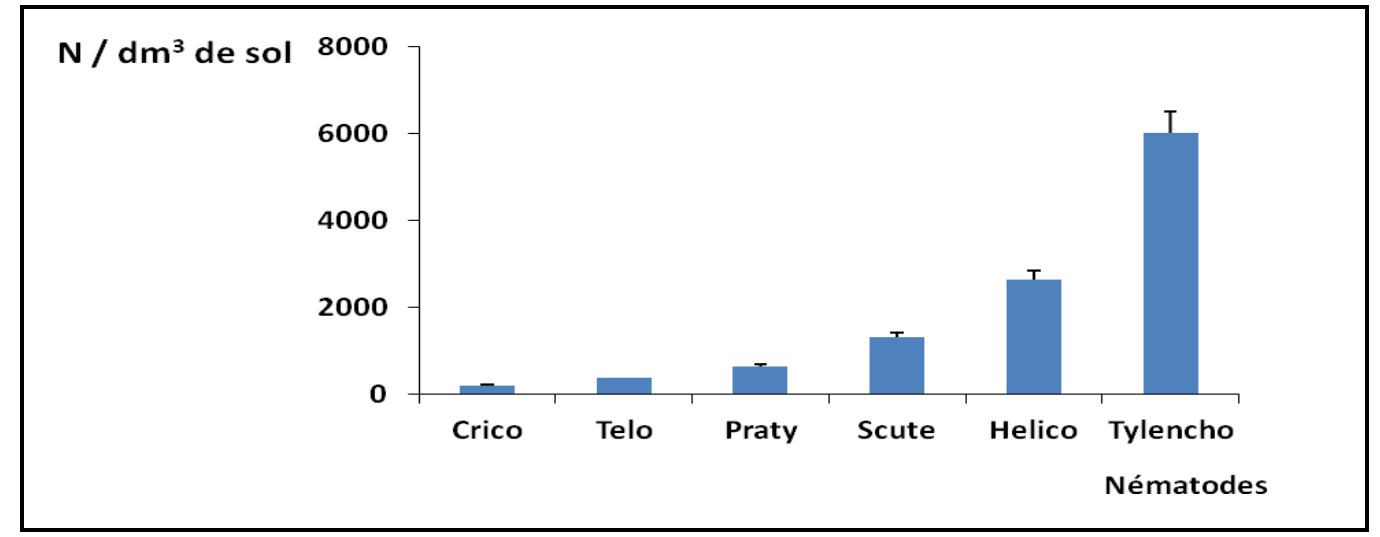

Praty $=$ P. brachyurus $;$ Tylencho $=$ T. martini $;$ Hélico $=H$. multicinctus $;$ Scute $=S$. cavenessi $;$ Crico $=C$. curvatum $;$ Telo: T. indicus; Xiphi = Xiphinema sp

Figure 4 : Nématodes dans la jachère bordant le site.

\section{DISCUSSION}

Nématodes du sol

Les espèces de nématodes recensées sur le site et dans la jachère appartiennent aux trois groupes de nématodes phytoparasites: les parasites internes ou endoparasites $(P$. brachyurus), les parasites externes ou ectoparasites (T. martini, $X s p, T$. indicus et $C$. curvatum), les parasites mixtes ou semiendoparasites ( $S$. cavenessi et $H$. multicinctus). Parmi elles, T. martini et $P$. brachyurus ont été signalées comme des espèces liées à la culture du sorgho. En particulier, $P$. brachyurus est signalée comme 
un nématode omniprésent, fréquemment lié à la culture du sorgho (Motalaote et al., 1987 ; Mc Donald et Nicol, 2005 ; Talwana et al., 2008 ; Villenave et al., 2010). Nos résultats montrent que les taux d'infestation des différents nématodes varient d'une rotation à une autre. La monoculture du sorgho a été la rotation culturale la plus infestée et certains nématodes ( $P$. brachyurus, T. martini et $H$. multicinctus) ont fortement régressé d'une rotation à une autre. En particulier, $P$. brachyurus qui est un nématode abondant dans la monoculture de sorgho, disparaît sous les autres rotations. Cette observation confirme le statut d'espèce principale inféodée au sorgho de $P$. brachyurus. Cette différence de comportement de cette espèce par rapport à la rotation culturale pourrait s'expliquer par son mode de parasitisme. On peut émettre l'hypothèse selon laquelle $P$. brachyurus étant un endoparasite strict généralement associé au sorgho, ne peut survivre et se reproduire en abondance sans sa plante hôte. Ces observations corroborent celles faites par des travaux antérieurs sur le rôle de la rotation culturale dans le contrôle des nématodes (Bachelier, 1978 ; Cadet, 1998 ; Stoll, 2002 ; Bélair, 2005 ; Bado et al., 2011). Nos résultats montrent également des comportements différents des nématodes du sol par rapport au type de traitements appliqués. Les traitements T3 et T5 avec apport simultané de matières organiques exogènes (fumier) et une fertilisation minérale ont eu des effets dépressifs pour les populations de nématodes. Ce qui n'a pas été le cas du traitement $\mathrm{T} 2$ où la matière organique employée est la paille. Ce traitement constitue avec les traitements $\mathrm{T} 4$ et $\mathrm{T} 6$, où il est employé uniquement une fertilisation minérale, les traitements les plus infestés. Cet effet des matières organiques exogènes pourrait être dû à plusieurs facteurs: soit à des substances toxiques produites lors de la poursuite de la dégradation de la matière organique dans le sol, ou encore à un changement dans la population microbienne du sol avec l'apparition de microorganismes nématophages (Cadet, 1998; Stoll, 2002). Dans la jachère bordant le site, la nématofaune a été plus diversifiée avec une prédominance des espèces $T$. martini et $H$. multicinctus. L'espèce $P$. brachyurus est très faiblement présente dans la jachère, ce qui confirme son statut d'espèce liée à la culture du sorgho souligné. Les espèces qui sévissent dans les champs cultivés ont fortement régressé ou disparu dans la jachère et des espèces absentes dans les champs ont également apparu dans la jachère. Ces observations sont conformes aux résultats de travaux antérieurs qui ont montré une dynamique différente des populations des nématodes entre espaces non cultivés (jachères et forêts) et cultivés (Cadet, 1998 ; Nandjui et al., 2007; Bois et al., 2000). Pontanier et al. (2003) et Forge et al. (2009) soulignent que la disparition ou la forte régression de certains nématodes phytoparasites dans la jachère n'est pas toujours signe d'une baisse de diversité ; mais il peut s'agir aussi d'une réorganisation spécifique. Nos résultats montrent que la rotation culturale couplée à l'apport des matières organiques et des fumures minérales permet de limiter l'impact des nématodes sur les terres agricoles.

\section{Nématodes des racines}

L'infestation des racines a été l'œuvre du seul nématode $P$. brachyurus. Nos observations montrent que la rotation culturale combinée à l'emploi des matières organiques a permis de contrôler la pénétration des nématodes dans les racines. Dans la monoculture de sorgho, les racines des plantes sont plus infestées par les nématodes que dans les deux autres rotations. Les rotations sorghoniébé et sorgho-coton ont offert une meilleure résistance des racines aux nématodes par rapport à la monoculture. Le principe de base 
de l'assolement qui est de séparer le ravageur de sa plante-hôte dans l'espace et dans le temps pour limiter des actions néfastes du ravageur est confirmé par nos résultats. Pour Stoll (2002), l'introduction par la rotation de plantes non-hôtes à un ravageur permet d'interrompre le cycle biologique de celui-ci et réduit la probabilité d'infestation des cultures suivantes. L'influence de la rotation pour le contrôle des nématodes a été démontrée dans de nombreux travaux antérieurs (Cadet, 1998; Bélair, 2005 ; Talwana et al., 2008 ; Bado et al., 2011). En particulier, il a été démontré que la culture du sorgho en rotation comportant une légumineuse était moins infectée par les nématodes (Bado, 2002). La rotation comportant une légumineuse mobilise plus d'azote pour la culture suivante. Cette mobilisation d'azote se fait par le processus de la fixation symbiotique qui permet aux légumineuses de mobiliser plus d'azote que les autres cultures. L'azote apporté à la plante se retrouve dans la zone du chevelu racinaire, améliore la possibilité d'absorption des racines et empêche les nématodes de pénétrer dans la plante (Sarah, 1991; Mateille, 1994 ; Dmowska et al., 1995). Nos résultats montrent aussi qu'au sein de chaque type de rotation, les traitements apportant la matière organique (fumier) et une fertilisation minérale (T3 et $\mathrm{T} 5$ ) sont les traitements où les racines du sorgho sont moins infestées par les racines. Les taux d'infestation plus élevés des autres traitements par rapport à ces deux traitements montrent que c'est la combinaison matière organique et minérale qui permet de mieux résister aux parasites. Nos résultats sont conformes à ceux de nombreux auteurs qui ont montré que l'apport de matière organique augmente les stocks organiques du sol et confère aux plantes plus de vigueur pour résister aux nématodes (Sarah, 1991; Mateille, 1994 ; Dmowska et al., 1995; Stoll, 2002; Bélair, 2005; Forge et al., 2009).

\section{Conclusion}

Cette étude a identifié les nématodes liés à la culture du sorgho dans le sol et dans les racines. Les espèces de nématodes identifiées ont montré des comportements divers par rapport aux pratiques culturales. Les apports de matières organiques exogènes, la fertilisation minérale et les rotations culturales pratiquées, ont permis de contrôler l'infestation du sorgho par les nématodes, en particulier l'infestation par $P$. brachyurus et $T$. martini, les deux principales espèces qui lui sont inféodées. Ces résultats corroborent notre hypothèse selon laquelle les pratiques agricoles ont une influence sur les densités des populations de nématodes dans le sol et les racines. L'amélioration des conditions culturales a permis aux plantes de mieux se défendre face aux nématodes.

\section{REMERCIEMENTS}

Nous remercions les techniciens de recherche, Sanou Martin à la Station de Recherches Agricoles de Saria, et Kièmdé Salam au laboratoire de nématologie du Centre Régional de Recherches Environnementales et Agricoles de Bobo Dioulasso pour leur appui lors des travaux de terrain et d'extraction des nématodes. Nous remercions également Diallo Boukary Ousmane et Guissou Tibi, Chargés de Recherches à l'Institut de l'Environnement et de Recherches Agricoles pour leur appui dans le traitement statistique des données.

\section{REFERENCES BIBLIOGRAPHIQUES}

Bachelier G. 1978. La Faune du Sol, son Ecologie et son Action. OSRTOM: Paris.

Bado BV. 2002. Rôle des légumineuses sur la fertilité des sols ferrugineux tropicaux des zones guinéenne et soudanienne du Burkina Faso. Thèse de Doctorat, Université Laval, département des sols et de génie agroalimentaire, Québec, Canada, p. 197. 
Bado V, Sawadogo A, Thio B, Bationo A, Traoré K, Cescas M. 2011. Nematode infestation and N-effect of legumes on soil and crop yields in legume-sorghum rotations. Agricultural Sciences, 2(2): 4955.

Bélair G. 2005. Les nématodes, ces anguillules qui font suer les plantes par la racine. Phytoprotection, 86(1): 65-69.

Bois JF, Cadet P, Plenchette CH, Duponnois R. 2000. Impact des nématodes phytoparasites de la zone SoudanoSahélienne du Sénégal sur la croissance du mil en conditions contrôlées. Étude et Gestion des Sols, 7(4): 271-278.

Bonzi M. 2002. Évaluation et déterminisme du bilan de l'azote en sols cultivés du centre Burkina Faso: Étude par traçage isotopique $15 \mathrm{~N}$ au cours d'essais en station et en milieu paysan. Thèse de Doctorat, INPL, Nancy, p. 177.

Cadet P. 1998. Gestion écologique des nématodes phytoparasites tropicaux. Cahier d'Agriculture, 7: 187-194.

Celetti MJ. 2006. Échantillonnage du sol et des racines visant le dénombrement des nématodes phytoparasites. Fiche Technique, (ed) la Reine pour l'Ontario: Canada.

De Weale D, Jordan ME. 1988. Plant parasitic nematodes on field crops in South Africa. 2. Sorghum. Revue Nématol., 11(2): 203212.

FAO. 2010. FAOSTAT Agriculture. http://faostat.fao.org/site/567/ Desktop Default.aspx?PageID=567\#ancor.(26/05/ 11)

Forge TA, Kempler C. 2009. Organic mulches influence population densities of rootlesion nematodes, soil health indicators, and root growth of red raspberry. Canadian Journal of Plant Pathology, 31(2): 241-249.

Hien E. 2004. Dynamique du carbone dans un Acrisol ferrique du Centre Ouest Burkina: Influence des pratiques culturales sur le stock et la qualité de la matière organique. Thèse de Doctorat, Ecole Nationale Supérieure Agronomique de Montpellier, p. 140.

Lompo F. 2008. Effets induits des modes de gestion de la fertilité sur les états du phosphore et la solubililité des phosphates naturels dans deux sols du Burkina Faso. Thèse de Doctorat d'État ès-Sciences Naturelles, Université de Cocody, Côte d'Ivoire, p. 178.

Mc Donald HA, Nicol MJ. 2005. Nematodes Parasites of Cereals. In Plant Parasitic Nematodes in Subtropical and Tropical Agriculture (2nd edn), Luc M, Sikora RA, Bridge J (eds). Cabi Publishing: Wallingford, UK; 131-192.

Merny G, Luc M. 1969. Les techniques d'échantillonnages des peuplements de nématodes dans le sol. In Problèmes d'Écologie: l'Echantillonnage des Peuplements d'Animaux des Milieux Terrestres, Lamotte M, Bourlière F (eds). Masson \& Cie : Paris, France; 236-272.

Motalaote B, Starr JL, Frederiksen RA, Miller FR. 1987. Host status and susceptibility of sorghum to pratylenchus species. Revue Nématol., 10(1): 81-86.

Nandjui J, Gnonhouri GP, Tondoh EJ, Tano Y. 2007. Réponse des nématodes à la perturbation des forêts dans la région d'Oumé, Côte d'Ivoire. Sciences \& Nature, 4(2): 189-196.

Ouédraogo S. 2005. Intensification de l'agriculture dans le plateau central du Burkina Faso: Une Analyse des possibilités à partir des nouvelles Technologies. Thèse de PhD, Rijks Universiteit Groningen, p. 336.

Pontanier R, Floret C. 2003. Les indicateurs $\mathrm{du}$ fonctionnement et du changement $\mathrm{du}$ milieu rural. In Savanes Africaines : des Espaces en Mutation, des Acteurs face à de Nouveaux défis, Jamin JY, Seiny BL, Floret C (eds). Actes du colloque, 27-31 
mai 2002, Garoua, Cameroun. Cirad : Montpellier, France; 7-17

Prot JC. 1985. Importance des nématodes phytoparasites en zones Sahéliennes. Lettre d'Information, 13: 3-6.

Sedogo PM. 1993. Evolution des sols ferrugineux lessivés sous culture : incidence des modes de gestion sur la fertilité. Thèse de $3^{\text {ème }}$ cycle, Université Nationale de Côte d'Ivoire, p. 285.

Stoll G. 2002. Protection Naturelle des Végétaux en Zones Tropicales. Margraf Verlag: Allemagne.

Talwana HL, Butseye MM, Tusiime G. 2008. Occurrence of plant parasitic nematodes and factors that enhance population buildup in cereal-based cropping systems in Uganda. African Crop Science Journal, 16(2): 119-131.

Villenave C, Saj S, Pablo AL, Sall S, Djigal D, Pablo JL, Bonzi M. 2010. Influence of long-term organic and mineral fertilization on soil nematofauna when growing Sorghum bicolor in Burkina Faso. Biol. Fertil. Soil, 46: 659-670.

Weber A, Oerke EC, Dehne HW, Schonbeck F. 1994. Crop Production and Crop Protection: Estimated Losses in Major Food and Cash Crops. Elsevier Science: USA. 